\title{
Oxaliplatin but Not Irinotecan Impairs Posthepatectomy Liver Regeneration in a Murine Model
}

\author{
Perry A. Soriano, ${ }^{1}$ Nian Liu, ${ }^{2}$ Erick Castillo, ${ }^{1}$ Brock Foster, ${ }^{1}$ Avo Artinyan, ${ }^{1}$ Joseph Kim, ${ }^{1}$ \\ Wendong Huang, ${ }^{2}$ and Lawrence D. Wagman ${ }^{3}$
}

${ }^{1}$ Liver Tumor Program, City of Hope, 1500 E. Duarte Road, Duarte, CA 91010, USA

${ }^{2}$ Center for Gene Regulation and Drug Discovery, Beckman Research Institute, City of Hope, 1500 E. Duarte Road, Duarte, CA 91010, USA

${ }^{3}$ The Center for Cancer Prevention and Treatment, St. Joseph Hospital, 1000 West La Veta Avenue, Orange, CA 92868, USA

Correspondence should be addressed to Lawrence D. Wagman, lawrence.wagman@stjoe.org

Received 11 May 2011; Revised 1 September 2011; Accepted 7 September 2011

Academic Editor: Wolfram Trudo Knoefel

Copyright ( $) 2011$ Perry A. Soriano et al. This is an open access article distributed under the Creative Commons Attribution License, which permits unrestricted use, distribution, and reproduction in any medium, provided the original work is properly cited.

\begin{abstract}
Introduction. We examined the murine hepatectomy model of liver regeneration (LR) in the setting of neoadjuvant chemotherapy. Methods. C57BL/6 mice were randomized to receive neoadjuvant intraperitoneal (IP) injections of a control, oxaliplatin $(15 \mathrm{mg} / \mathrm{kg})$, or irinotecan $(100 \mathrm{mg} / \mathrm{Kg}$ or $250 \mathrm{mg} / \mathrm{Kg})$ solution. Hepatectomy $(70 \%)$ was performed 14 days after the final IP treatment. Animals were sacrificed at postoperative day (D) 0,1,2,3, and 7. Liver remnants and serum were collected for analysis. $T$-tests for independent samples were used for statistical comparisons. Results. For oxaliplatin, percent LR did not differ at D1 or D2 but was significantly less at D3 (89.0\% versus $70.0 \%, P=0.048)$ with no difference on D7 $(P=0.21)$. Irinotecan-treated mice at both dose levels $(100 \mathrm{mg} / \mathrm{Kg}$ and $250 \mathrm{mg} / \mathrm{Kg}$ ) showed no significant differences in LR. BrdU incorporation was significantly decreased in oxaliplatin-treated animals $(\mathrm{D} 1,2,3)$. Conclusions. Neoadjuvant oxaliplatin but not irinotecan impairs early LR in a posthepatectomy murine model which correlates with decreased DNA synthesis.
\end{abstract}

\section{Introduction}

2010 an estimated 142,570 people developed colorectal cancer (CRC) with an estimated 51,370 people dying of the disease [1]. Synchronous liver metastases are found in $20 \%$ of patients, and more than half of those diagnosed with CRC will go on to develop metachronous liver metastases $[2,3]$. Liver only or liver-predominant disease affects $20-35 \%$ of patients, affording those with resectable lesions the possibility of long-term survival. In selected cases with R0 resection, 10-year overall survival has been reported in the literature to range from $17-25 \%[4,5]$. In addition to its adjuvant use in Stage 3 colon cancer and following hepatic resection, chemotherapy has the potential to convert borderline or unresectable liver disease to resectable disease by reducing the size of the tumor to an amenable dimension. Furthermore, neoadjuvant chemotherapy has been advocated as a test for aggressive tumor biology [6-8].
Timing and appropriateness of chemotherapy, however, is debated, and there are concerns regarding worse outcomes in heavily treated patients [9]. In this regard, steatohepatitis, steatosis, and sinusoidal injury have been linked to the use of irinotecan, fluoropyrimidines and oxaliplatin [10].

Animal models for the study of posthepatectomy liver regeneration are well described [11]. These models have yet to be applied to the study of commonly used agents for CRC. Given first-line use of oxaliplatin and irinotecan for stage IV CRC, these agents were chosen for investigation. We hypothesized that posthepatectomy liver regeneration is impaired by oxaliplatin and/or irinotecan administration and that this impairment can be demonstrated in a mouse model.

\section{Materials and Methods}

2.1. Animal Maintenance and Treatments. Eight-week-old C57BL/6 male mice, weighing between 23-25 grams, were 
obtained from commercial sources (Taconic Farms, Hudson, NY). The animals were housed under standard 12-hour light/12-hour dark conditions with standard feed and water ad libitum. After a minimum of 48 hours acclimation, animals were randomized to receive either oxaliplatin (15 mg/Kg), irinotecan (100 mg/kg or $250 \mathrm{mg} / \mathrm{kg}$ ) or control solution (dextrose $5 \%$ water) by intraperitoneal injection. Animal tolerance of chemotherapy was closely monitored, and posthepatectomy animals were evaluated daily. Animal handling, drug administration, monitoring, and survival surgery protocols were approved by the City of Hope, Research Animal Care Committee.

2.2. Chemotherapy. Oxaliplatin and irinotecan were obtained through the City of Hope, Investigational Drug Services and diluted in non-chloride-containing solution (dextrose $5 \%$ water) to deliver the determined dose in an approximate volume of $100 \mathrm{mcL}$. Dose regimens were based on data from in vivo activity in previously described colon cancer tumor models in mice $[12,13]$. Oxaliplatin $15 \mathrm{mg} / \mathrm{Kg}$ was administered IP $\times 1$ dose. Irinotecan was administered at two dose levels as follows: regimen $\mathrm{A}, 100 \mathrm{mg} / \mathrm{Kg}$, IP divided in 2 weekly doses and regimen B, $250 \mathrm{mg} / \mathrm{Kg}$ IP divided in 3 weekly doses $(75 \mathrm{mg} / \mathrm{Kg}, 75 \mathrm{mg} / \mathrm{Kg}, 100 \mathrm{mg} / \mathrm{Kg})$. Fourteen days after the last control or chemotherapy injection, a $70 \%$ hepatectomy was performed. Despite using well-established dosing schedule [12] in a dedicated vivarium with skilled personnel, 19 of 32 animals died from the initial treatment with oxaliplatin. There was no mortality in the irinotecan group. All surviving animals were included in the surgical portion of the experiment.

2.3. Animal Surgery. The left and median lobes were resected with preservation of the gallbladder for 70\% hepatectomy. Briefly, tribromoethanol (Avertin) anesthetic was administered IP $(250 \mathrm{mg} / \mathrm{Kg})$. After sterile prep a subxiphoid transverse incision was created and the median and left liver lobes were exteriorized. The lobes were encircled with silk ligature, their vascular pedicles tied at the base and the lobes resected. Care was taken to spare the gallbladder and associated bile ducts. Closure was accomplished with autoclips. Buprenorphine was administered $(0.5 \mathrm{mg} / \mathrm{Kg}$ subcutaneously) upon awakening. At postoperative days 0, $1,2,3$, and 7 , remnant right and caudate lobes were harvested, and blood was collected from the retroorbital sinus concomitant with animal sacrifice. In the oxaliplatin experimented cohort, there were 3 perioperative deaths (2 oxaliplatin treated, 1 control). There was no mortality in the irinotecan cohort.

2.4. Percent Liver Regeneration by Mass. Percent liver regrowth was calculated by the following formula: (Mass of regenerating liver remnant in grams) $\div$ (Mass of resected liver lobes in grams $) /(0.7) \times 100$.

2.5. Liver Histology and Bromodeoxyuridine (BrdU) Incorporation. In vivo BrdU staining was accomplished by intraperitoneal injection of $\mathrm{BrdU}(100 \mathrm{mg} / \mathrm{Kg}) 2$ hours prior

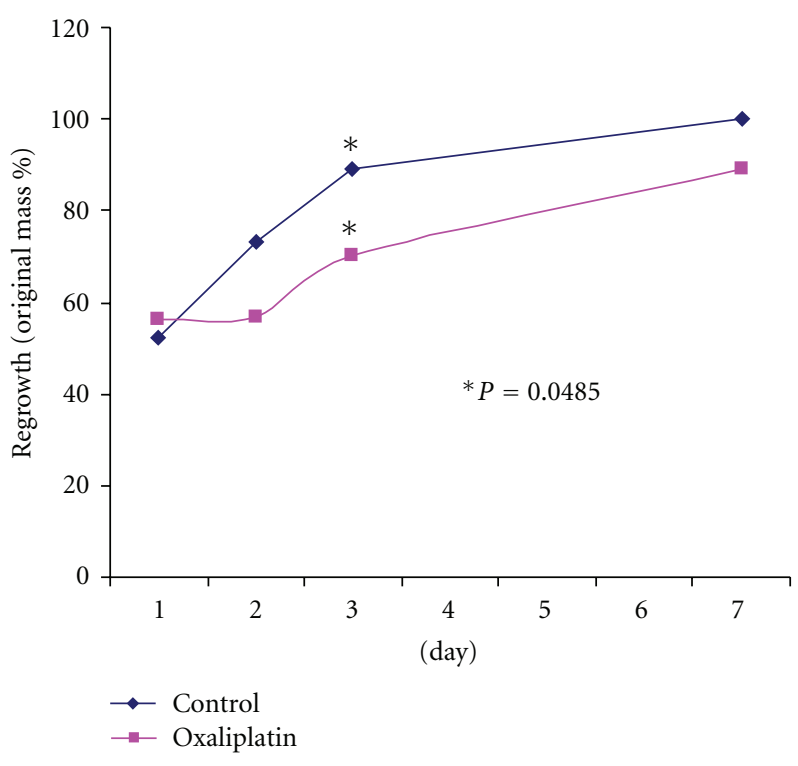

FIGURE 1: Liver regeneration by percent regrowth, oxaliplatin $15 \mathrm{mg} / \mathrm{Kg}$ versus control, differs significantly at day 3 .

to sacrifice. Uniform samples of hepatic parenchyma were removed and fixed in 4\% formaldehyde solution, embedded in paraffin, sectioned at 5 micrometers, and stained with hematoxylin and eosin. BrdU immunohistochemical staining was performed using a commercially available kit (Roche). The number of positively stained nuclei was counted in 3 randomly selected high-power fields per sample, one sample from at least 2 mice per time point and arm.

2.6. ALT Analysis. Under anesthesia prior to sacrifice, approximately $500 \mathrm{mcL}$ of blood was drawn from the retroorbital sinus and placed in serum separator tubes (Falcon). Collected serum was then analyzed for ALT after 10-fold dilution in $7 \%$ bovine serum albumin.

2.7. Statistical Analysis. Statistical comparisons were performed using $t$-tests for independent samples.

\section{Results}

3.1. Oxaliplatin. 22 animals underwent $70 \%$ hepatectomy in the oxaliplatin versus control study, 9 animals in the control arm, and 13 in the oxaliplatin arm. Animal weights of the survivors were similar to those of the control group at the time of hepatectomy. There were 3 perioperative deaths; 1 in the control arm and 2 in the oxaliplatin arm which were technical in nature (pneumothorax, excessive manipulation of lobes on extraction, and hemorrhage).

Percent liver regrowth (Table 1 and Figure 1) at day 1 following hepatectomy did not differ between oxaliplatintreated and control mice $(56.1 \%$ versus $52.5 \%$, resp., $P=$ 0.312 ). Data collected on day 2 suggests less regrowth in the oxaliplatin-treated arm (57.6\% versus $73.0 \%, P=0.154)$; however this was not statistically significant. Regeneration was significantly less in the treatment arm at day $3(70.0 \%$ 
TABLE 1: Liver regeneration after oxaliplatin treatment, percent regrowth by mass, days 1, 2, 3, and 7.

\begin{tabular}{lccc}
\hline Day & Regrowth control & Regrowth oxaliplatin & Independent $T$-test \\
\hline Day 1 & $56.1 \%(N=2)$ & $52.5 \%(N=3)$ & $P=0.312$ \\
Day 2 & $73.0 \%(N=2)$ & $57.6 \%(N=3)$ & $P=0.154$ \\
Day 3 & $89.0 \%(N=2)$ & $70.0 \%(N=3)$ & $P=0.048$ \\
Day 7 & $99.0 \%(N=2)$ & $89.8 \%(N=2)$ & $P=0.214$ \\
\hline
\end{tabular}

$N$, number of animals per group.

versus $89.0 \%, P=0.048$ ). By 7 days following hepatectomy, delayed LR in the oxaliplatin-treated arm was no longer found to be statically significant $(89.8 \%$ versus $99.0 \%, P=$ $0.214)$.

Hepatocyte injury was assessed by measurement of ALT levels. ALT levels peaked at posthepatectomy day 1 and normalize by day 3 . ALT levels in oxaliplatin-treated animals were not found to be statistically different than controls throughout the study (Figure 5).

BrdU incorporation was used to determine if oxaliplatin impairs DNA synthesis (cellular division), thus contributing to impaired liver regrowth (Figure 3). DNA synthesis was significantly higher in the control arm at all three measured timepoints. Oxaliplatin-treated animals showed significantly less incorporation consistent with reduced DNA synthesis.

\subsection{Irinotecan. In the irinotecan experiments no animals} experienced chemotherapy-related mortality. Weights were similar between groups at the time of hepatectomy. Neither dose level, group A $(100 \mathrm{mg} / \mathrm{Kg}, N=15$; control $N=8)$ nor group B $(250 \mathrm{mg} / \mathrm{Kg}, N=17$; control $N=5)$ showed significant impairment in liver regrowth by mass compared with respective controls (Figure 2). Similar to the oxaliplatin group, irinotecan-treated animals showed peak ALT levels at day 1 with return to baseline between days 3-7 (data not shown). In contrast to the oxaliplatin results, BRDU incorporation in irinotecan-treated animals was similar or increased compared to controls (Figure 4).

3.3. Histology. Histologic examination of regenerating liver specimens showed no evidence of hepatic sinusoidal obstruction in oxaliplatin-or-irinotecan treated animals. Mild ballooning changes due to increased cytoplasmic water were seen in both treated and untreated groups. In the oxaliplatin arm, mild portal inflammation with necrosis near the portal triads and microvesicular steatosis were seen in two animals, one at posthepatectomy day 2 and one at day 3 .

\section{Discussion}

The liver's remarkable ability to restore a functionally adequate portion of its previous volume following surgical resection is tightly regulated by mechanisms that include bile acid interactions with the FXR nuclear receptor and several other complex mechanisms $[11,14]$. The mouse liver regeneration model is well described and highly reproducible in this posthepatectomy setting. The differences in regeneration are demonstrated at early timepoints, namely, days 2 and 3 after hepatectomy [11]. We chose to apply this model to the study of liver regeneration after treatment with commonly used modern chemotherapeutic agents for CRC. The oxaliplatin dose was selected based on established, species-specific doses from the research literature [12]. An unexpected toxicity (mortality) was observed in this experiment. The animal deaths affected the group sizes, but only impacted the planned experimental animal numbers (approved by the Research Animal Care Committee) that would be required to achieve definitive statistical results in one cohort.

We discovered that oxaliplatin-treated animals showed significantly reduced regrowth on the third posthepatectomy day. This finding has not been previously described in a preclinical model and may, in part, be due to the mechanism of oxaliplatin cytotoxicity. Oxaliplatin is a third-generation platinum derivative that acts at the level of DNA by forming bulky DNA adducts [15]. Most commonly, intrastrand links between guanine and adenine are formed by the platinum moiety. DNA synthesis is impaired by these adducts which in turn leads to strand breaks and subsequent apoptosis. Oxaliplatin's mechanism of action is consistent with the marked decrease in DNA synthesis demonstrated by the decrease in BrdU staining in these experiments. BrdU staining was more sensitive than percent regrowth by weight. Analysis of oxaliplatin's impact on BrdU staining were demonstrated with significant differences as early as day 1 following hepatectomy with significant impairment in DNA synthesis continuing through day 3 . These data combined with the absence of direct hepatic damage, as evidenced by nonsignificant differences in ALT, suggest oxaliplatin blunts LR by inhibiting cell division in the early postoperative period. However, this effect appears to be lost by 7 days postoperatively as physiologic mechanisms to restore appropriate liver function normalize liver size by this time. Despite the consistency (growth and DNA synthetic activity) of these data, the small number of experimental animals requires they be viewed as exploratory, not definitive. The experimental model is well established, and the BrdU incorporation is a sensitive measure of DNA synthesis. The current literature contains variable conclusions on the impact of chemotherapy on liver regeneration. In part, this is due to differences in experimental modeling (e.g., number of chemotherapy injections, use of Ki-67, and single time-point analysis). The current series of experiments provides sequential time point evaluation at $0,1,2,3$, and 7 days in an attempt to mimic the immediate, early, and longer phases of hepatic regeneration in the human. This sequential reporting is unique in investigations of this type. This topic area remains controversial, and additional experiments with 


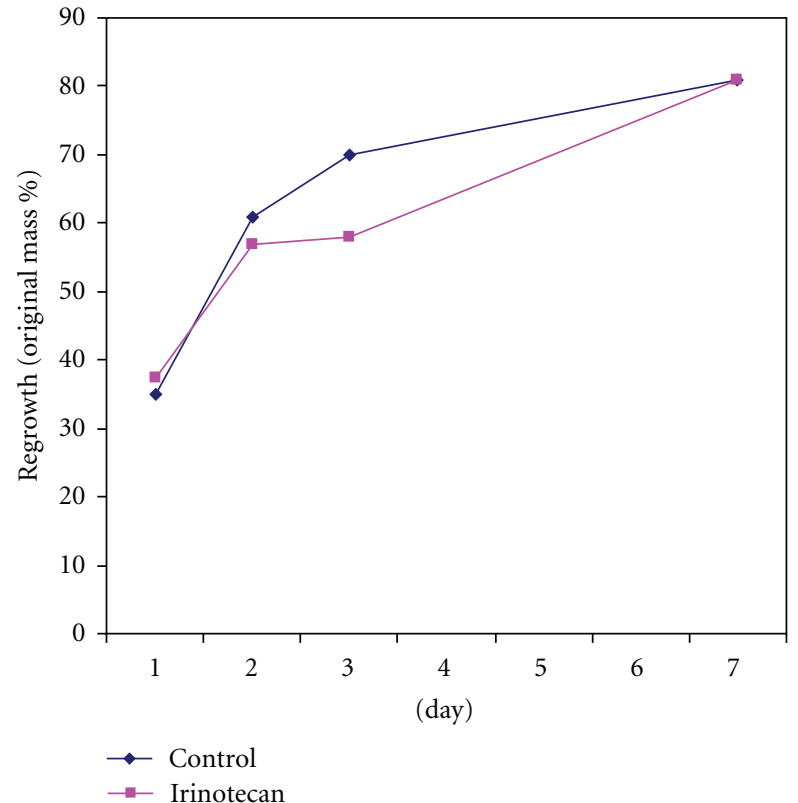

(a)

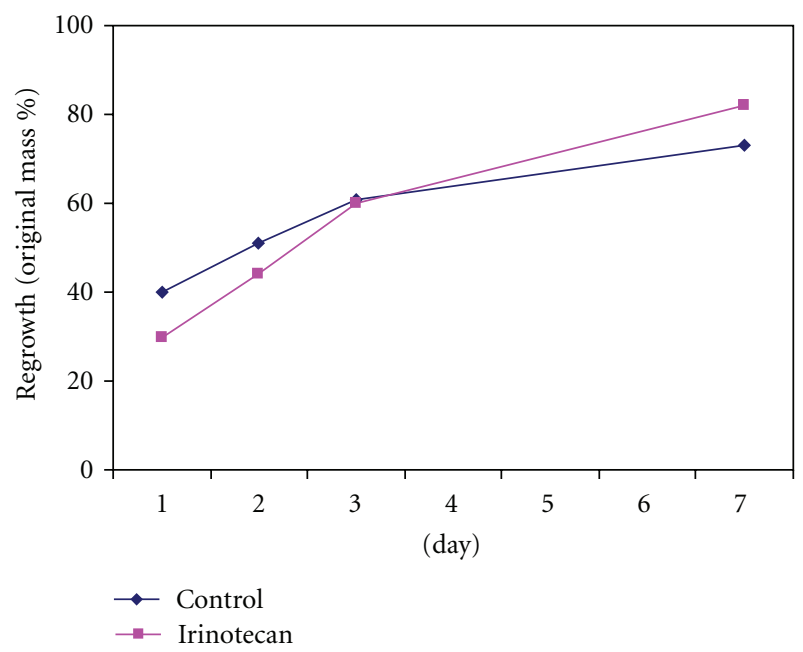

(b)

Figure 2: Liver regeneration by percent regrowth does not differ after irinotecan treatment at cumulative $100 \mathrm{mg} / \mathrm{Kg}$ (a) and $250 \mathrm{mg} / \mathrm{Kg}$ (b) dose levels.

a consistent experimental model will be the most definitive way to answer the controversies and variability in results.

Irinotecan-treated animals did not show differences in liver regrowth despite treatment at previously documented pharmacologically active doses $[16,17]$. BrdU incorporation assays corroborate these findings with no decrease but rather, a nonstatistically significant increase in DNA synthesis. Increased DNA synthesis with irinotecan treatment may be related to the drug's mechanism of action. Irinotecan inhibits topoisomerase I, stabilizes single-strand breaks and results in double-strand breakage though interaction at the replication fork [18]. Our results suggest that the structure and coiling of DNA is altered by irinotecan without direct effect on the

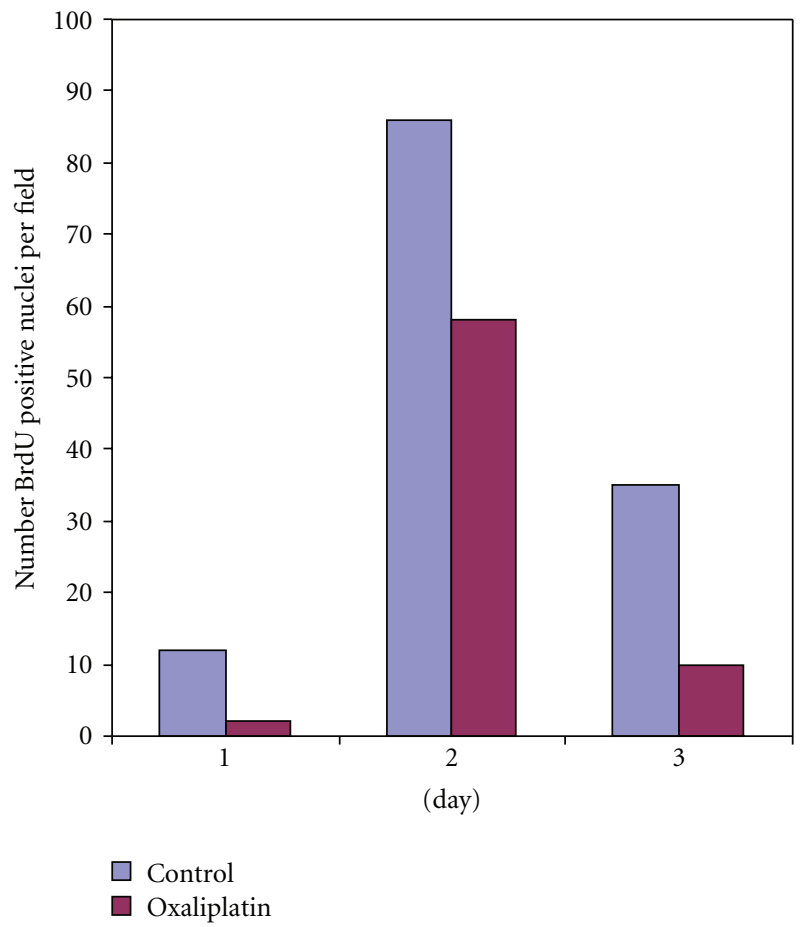

FIGURE 3: BrdU incorporation is significantly less in oxaliplatin$(15 \mathrm{mg} / \mathrm{kg})$ treated animals at days 1,2 , and 3 .

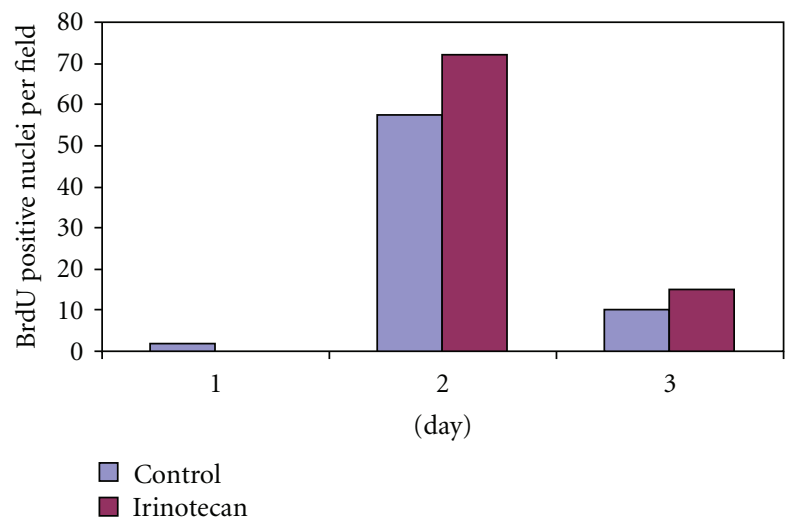

Figure 4: BrdU incorporation is increased in irinotecan$(100 \mathrm{mg} / \mathrm{kg})$ treated animals.

cells ability to synthesize DNA and thus incorporate BrdU. Despite the somewhat counterintuitive nature of this finding, a higher proportion of cells in S-phase after irinotecan treatment has been described in animal and clinical settings [19].

High toxicity was seen in the animals receiving oxaliplatin. This occurred despite the use of previously reported doses [20]. In our experiments, although the toxicity was high during the administration of oxaliplatin, the surviving animals were fully recovered prior to hepatectomy with no difference in animal weight or appearance in the oxaliplatintreated animals when compared to irinotecan-treated animals. This argues that differences in regrowth were liver 


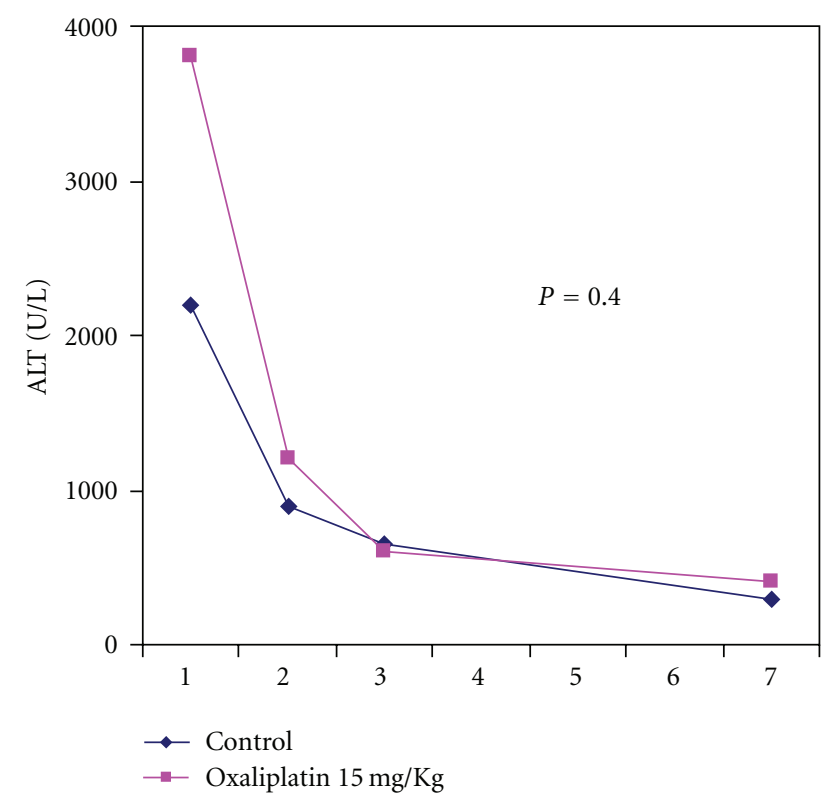

FIGURE 5: Representative plot of ALT levels showing a peak at day 1 with normalization between day 3 and 7 .

specific and not a byproduct of other factors such as a weakened state or poor nutrition.

Recently, clinical studies have raised concerns regarding the significant hepatotoxicity of chemotherapeutic agents for CRC [21]. Oxaliplatin is implicated in the "blue liver" syndrome from hepatic sinusoidal obstruction, and worse posthepatectomy outcome is reported in association with chemotherapy-related steatohepatitis primarily with irinotecan $[22,23]$. Histologic examination of the liver in 2 animals showed microvesicular steatosis and mild periportal inflammation. However, this was an uncommon finding. The differences seen in regeneration and DNA synthesis, therefore, likely reflect changes not yet evident on H\&E, but in part detectable with special staining techniques such as BrdU immunohistochemistry.

Clinical guidelines for hepatectomy recommend more conservative volumes of liver resection in chemotherapytreated patients with a goal future liver remnant of $30 \%$, rather than $20 \%[16,24]$. Given the adverse effects of chemotherapy on the liver, our goal was to establish an animal model to study these interactions. We have shown early impairment of regenerative ability in oxaliplatin-treated animals. These findings are corroborated by decreased DNA synthesis. These data suggest that early in the patient's postoperative course, when the risk for liver failure is higher, regenerative mechanisms may be impaired. Future studies with this model will aim at abrogating these effects.

\section{Conclusion}

The mouse $70 \%$ hepatectomy model provides a useful tool for studying the effects of chemotherapy on posthepatectomy liver regeneration. We demonstrate that oxaliplatin impairs early liver regeneration in a posthepatectomy model and that this reduced regrowth correlates with decreased DNA synthesis. Conversely, irinotecan did not impair regeneration or DNA synthesis.

\section{Acknowledgments}

The authors wish to thank members of the Department of Pathology at City of Hope, namely, Sofia Loera and her team for expert performance of the BrdU IHC staining, and Dr. Peiguo Chu, MD, for prompt and thorough histologic review.

\section{References}

[1] N. Howlader, A. M. Noone, M. Krapcho et al., "SEER Cancer Statistics Review, 1975-2008," National Cancer Institute. Bethesda, Md, USA, 2011, http://seer.cancer.gov/csr/ 1975_2008/.

[2] L. Weiss, E. Grundmann, and J. Torhorst, "Haematogenous metastatic patterns in colonic carcinoma: an analysis of 1541 necropsies," Journal of Pathology, vol. 150, no. 3, pp. 195-203, 1986.

[3] N. Kemeny, Y. Huang, A. M. Cohen et al., "Hepatic arterial infusion of chemotherapy after resection of hepatic metastases from colorectal cancer," New England Journal of Medicine, vol. 341, no. 27, pp. 2039-2048, 1999.

[4] M. M. Borner, "Neoadjuvant chemotherapy for unresectable liver metastases of colorectal cancer-too good to be true?" Annals of Oncology, vol. 10, no. 6, pp. 623-626, 1999.

[5] J. S. Tomlinson, W. R. Jarnagin, R. P. DeMatteo et al., "Actual 10 -year survival after resection of colorectal liver metastases defines cure," Journal of Clinical Oncology, vol. 25, no. 29, pp. 4575-4580, 2007.

[6] G. Masi, S. Cupini, L. Marcucci et al., "Treatment with 5fluorouracil/folinic acid, oxaliplatin, and irinotecan enables surgical resection of metastases in patients with initially unresectable metastatic colorectal cancer," Annals of Surgical Oncology, vol. 13, no. 1, pp. 58-65, 2006.

[7] R. Adam, E. Avisar, A. Ariche et al., "Five-year survival following hepatic resection after neoadjuvant therapy for nonresectable colorectal [liver] metastases," Annals of Surgical Oncology, vol. 8, no. 4, pp. 347-353, 2001.

[8] T. M. Pawlik, R. D. Schulick, and M. A. Choti, "Expanding criteria for resectability of colorectal liver metastases," Oncologist, vol. 13, no. 1, pp. 51-64, 2008.

[9] J. N. Vauthey, T. M. Pawlik, D. Ribero et al., "Chemotherapy regimen predicts steatohepatitis and an increase in 90-day mortality after surgery for hepatic colorectal metastases," Journal of Clinical Oncology, vol. 24, no. 13, pp. 2065-2072, 2006.

[10] J. M. Cleary, K. T. Tanabe, G. Y. Lauwers, and A. X. Zhu, "Hepatic toxicities associated with the use of preoperative systemic therapy in patients with metastatic colorectal adenocarcinoma to the liver," Oncologist, vol. 14, no. 11, pp. 1095-1105, 2009.

[11] W. Huang, K. Ma, J. Zhang et al., "Nuclear receptor-dependent bile acid signaling is required for normal liver regeneration," Science, vol. 312, no. 5771, pp. 233-236, 2006.

[12] S. Cao, F. A. Durrani, and Y. M. Rustum, "Selective modulation of the therapeutic efficacy of anticancer drugs by selenium containing compounds against human tumor xenografts," Clinical Cancer Research, vol. 10, no. 7, pp. 2561-2569, 2004. 
[13] W. J. M. Jansen, B. Zwart, S. T. M. Hulscher, G. Giaccone, H. M. Pinedo, and E. Boven, "CPT-11 in human colon-cancer cell lines and xenograft: characterization of cellular sensitivity determinants," International Journal of Cancer, vol. 70, no. 3, pp. 335-340, 1997.

[14] P. A. Clavien, H. Petrowsky, M. L. DeOliveira, and R. Graf, "Strategies for safer liver surgery and partial liver transplantation," New England Journal of Medicine, vol. 356, no. 15, pp. 1545-1559, 2007.

[15] J. C. Byrd, I. W. Flinn, and M. R. Grever, "Introduction: chronic lymphocytic leukemia," Seminars in oncology, vol. 25, no. 1, pp. 4-5, 1998.

[16] W. J. M. Jansen, B. Zwart, S. T. M. Hulscher, G. Giaccone, H. M. Pinedo, and E. Boven, "CPT-11 in human colon-cancer cell lines and xenograft: characterization of cellular sensitivity determinants," International Journal of Cancer, vol. 70, no. 3, pp. 335-340, 1997.

[17] H. Shinohara, J. J. Killion, H. Kuniyasu, R. Kumar, and I. J. Fidler, "Prevention of intestinal toxic effects and intensification of Irinotecan's therapeutic efficacy against murine colon cancer liver metastases by oral administration of the lipopeptide JBT 3002," Clinical Cancer Research, vol. 4, no. 9, pp. 2053-2063, 1998.

[18] L. Iyer and M. J. Ratain, "Clinical pharmacology of camptothecins," Cancer Chemotherapy and Pharmacology, vol. 42, supplement, pp. S31-S43, 1998.

[19] N. Ramnath, N. Khushalani, K. Toth et al., "S-phase modulation by irinotecan: pilot studies in advanced solid tumors," Cancer Chemotherapy and Pharmacology, vol. 56, no. 5, pp. 447-454, 2005.

[20] G. Tortora, F. Ciardiello, V. Damiano et al., "Preclinical and phase I study of oxaliplatin and topotecan in combination in human cancer," Annals of Oncology, vol. 13, no. 3, pp. 392-398, 2002.

[21] A. Artinyan, E. Castillo, B. Foster et al., "Evaluation of the potential association of preoperative chemotherapy with steatohepatitis in surgical patients with hepatic colorectal metastases," Journal of Clinical Oncology, vol. 27, no. 15, supplement, 2009.

[22] J. N. Vauthey, T. M. Pawlik, D. Ribero et al., "Chemotherapy regimen predicts steatohepatitis and an increase in 90-day mortality after surgery for hepatic colorectal metastases," Journal of Clinical Oncology, vol. 24, no. 13, pp. 2065-2072, 2006.

[23] L. Rubbia-Brandt, V. Audard, P. Sartoretti et al., "Severe hepatic sinusoidal obstruction associated with oxaliplatinbased chemotherapy in patients with metastatic colorectal cancer," Annals of Oncology, vol. 15, no. 3, pp. 460-466, 2004.

[24] T. M. Pawlik, R. D. Schulick, and M. A. Choti, "Expanding criteria for resectability of colorectal liver metastases," Oncologist, vol. 13, no. 1, pp. 51-64, 2008. 


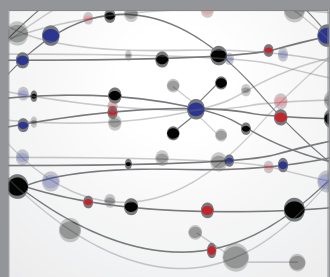

The Scientific World Journal
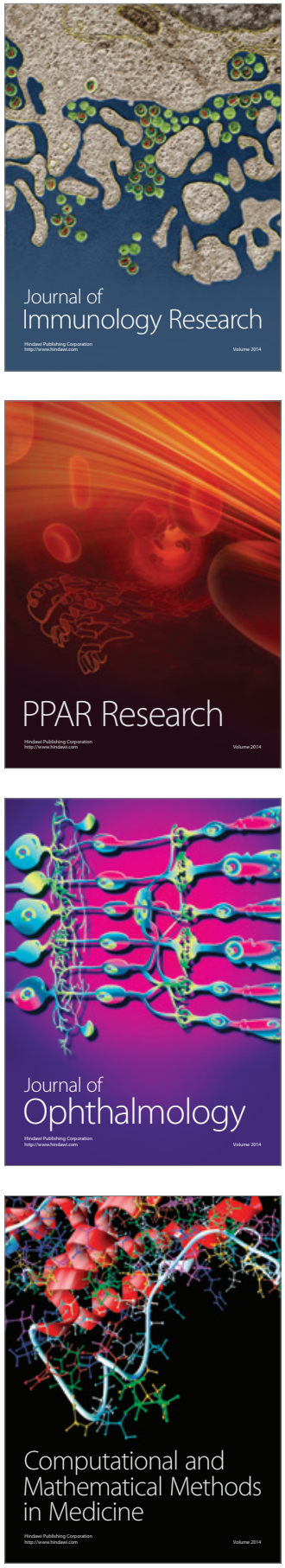

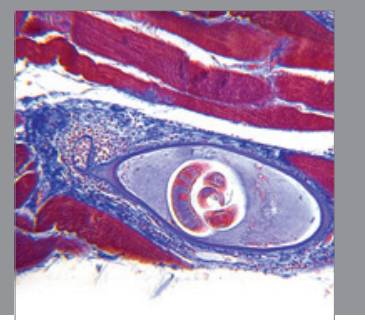

Gastroenterology

Research and Practice
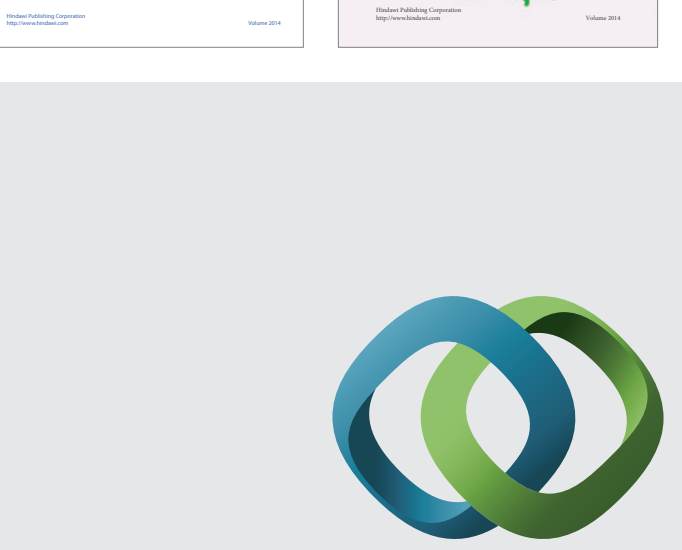

\section{Hindawi}

Submit your manuscripts at

http://www.hindawi.com
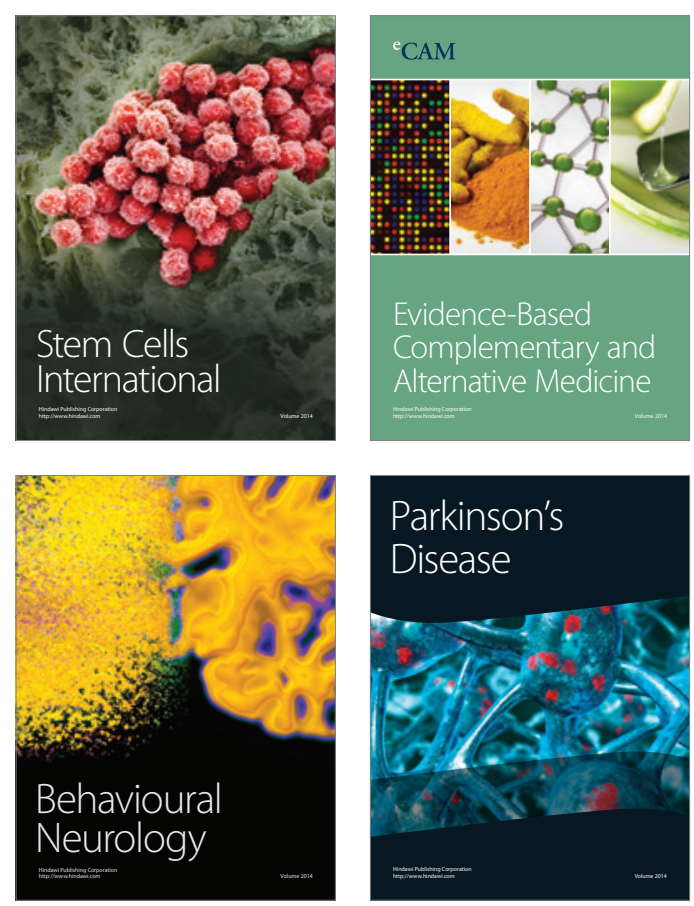

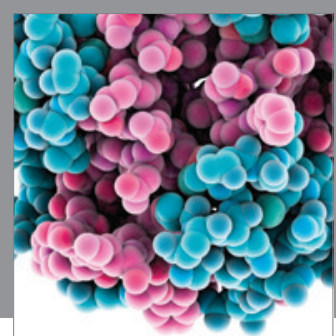

Journal of
Diabetes Research

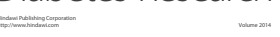

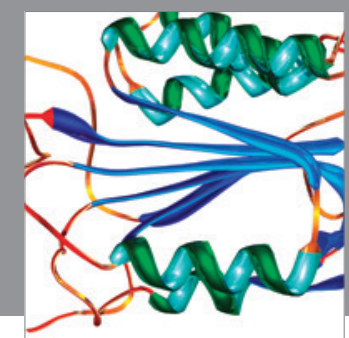

Disease Markers
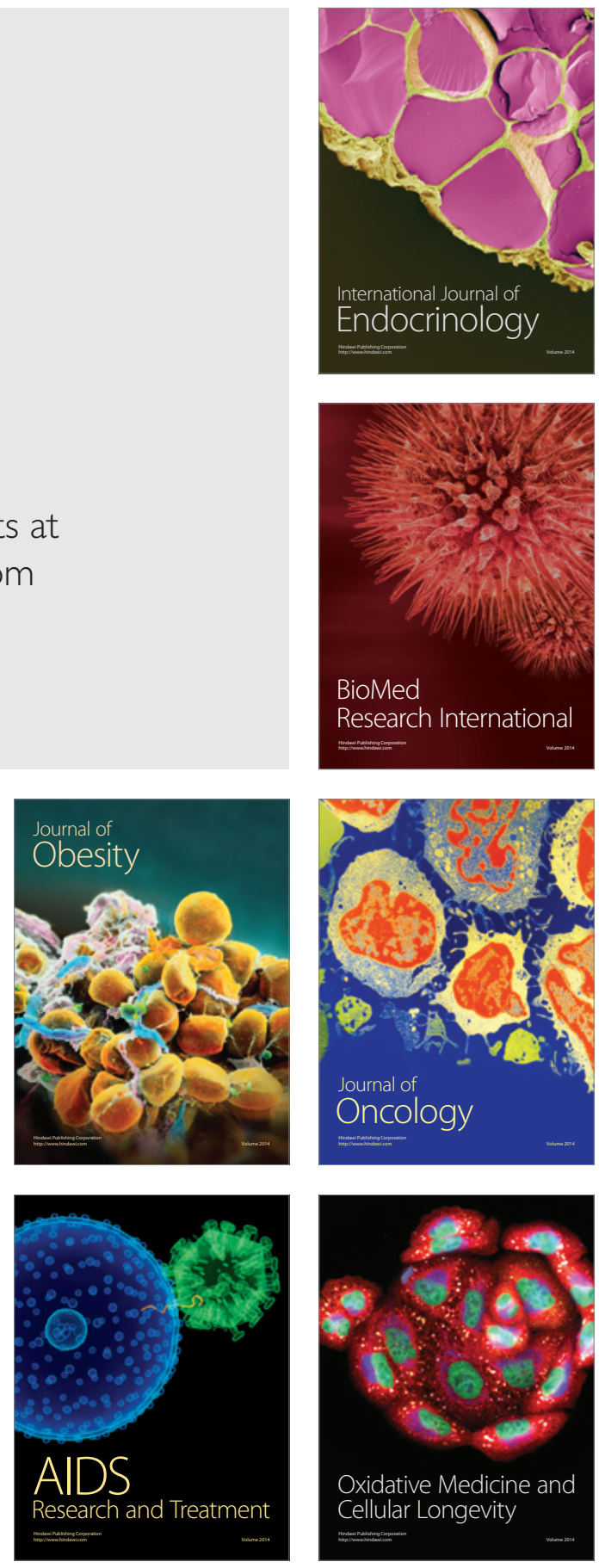